\title{
ACESSIBILIDADE EM REPOSITÓRIOS DIGITAIS BRASILEIROS
}

\section{ACCESSIBILITY IN BRAZILIAN DIGITAL REPOSITORIES}

\author{
Pietra Gomes Ramires \\ Graduanda em Biblioteconomia - FURG \\ pietragomes.gr@gmail.com \\ Brenda Costa Fonseca \\ Graduanda em Biblioteconomia - FURG \\ brenda07.fonseca@gmail.com \\ Tainã da Silva Rodrigues \\ Graduando em Biblioteconomia - FURG \\ 790.rodrigues@gmail.com \\ Maria de Fatima Santos Maia \\ Doutora em Comunicação e Informação \\ Professora do Curso de Biblioteconomia da FURG \\ mafas.maia@gmail.com
}

Resumo

Foi avaliado o grau de acessibilidade dos repositórios digitais brasileiros. Os dados foram coletados no website do Instituto Brasileiro de Informação em Ciência e Tecnologia (IBICT) e analisados com a ferramenta AccessMonitor. Os resultados mostraram que os repositórios brasileiros precisam dar mais atenção para a acessibilidade. O Repositório Eletrônico Institucional da Universidade Federal da Paraíba foi o repositório com maior grau de inclusão e CBPF Index foi o que alcançou o pior. Concluiu-se que o pressuposto de que repositórios são ambientes abertos e acessíveis não corresponde com a realidade. A maioria dos repositórios brasileiros não estão satisfatoriamente alinhados com as Diretrizes de Acessibilidade para Conteúdo Web (WCAG).

Palavras-chave: Acessibilidade digital; Repositórios Digitais; Inclusão digital; AccessMonitor.

\begin{abstract}
The degree of accessibility of Brazilian digital repositories was evaluated. The data were collected on the website of the Brazilian Institute of Information in Science and Technology (IBICT) and analyzed with the AccessMonitor tool. The results showed that Brazilian repositories need to pay more attention to accessibility. The Institutional Electronic Repository of the Federal University of Paraiba was the repository with the highest degree of inclusion and CBPF Index was the one that achieved the worst. It was concluded that the idea that repositories should be open and accessible environments does not correspond with reality. Most Brazilian repositories are not satisfactorily aligned with the Accessibility Guidelines for Web Content (WCAG).
\end{abstract}

Keywords: Digital accessibility; Digital repositories; Digital inclusion; AccessMonitor.

\section{INTRODUÇÃO}

Os ambientes informacionais digitais têm crescido de maneira acelerada e contínua, permitindo que a sociedade, de maneira geral, tenha maior acesso a toda espécie de informação (HOTT; CRUZ-RIASCOS, 2018). No entanto, este acesso parece não ser equânime, 
especialmente quando se trata de pessoas com deficiência, que necessitam de ferramentas específicas para utilizar plenamente os ambientes informacionais digitais. Muitas vezes, os preconceitos e rejeições já existentes no mundo analógico simplesmente são transferidos para o mundo digital, excluindo os indivíduos com algum tipo de deficiência de acessarem informações (BERRÍO-ZAPATA; SANTOS; CHALHUB, 2020). Portanto, muitos desafios ainda precisam ser ultrapassados para remover as barreiras de acesso aos ambientes digitais para pessoas com limitações físicas, motoras e/ou psíquicas.

Para Souza e Guimarães (2015), projetos de sistemas informacionais digitais precisam considerar a importância da disponibilização de recursos para todos os usuários, sem exceções. Neste contexto, a inclusão digital é peça essencial, pois grande parte das informações disponíveis atualmente estão armazenadas na internet (ANDRADE; LUCAS; NASCIMENTO, 2015). Sendo assim, as atividades de unidades e sistemas de informação devem ser norteadas pela permanente atenção sobre o perfil dos seus usuários e suas necessidades, adequando seus ambientes físicos e virtuais.

Neste cenário, encontram-se os repositórios digitais, que partem da ideia de que informações devem ser acessíveis e abertas, isto é, sem restrições, por conseguinte devem ser sistemas com acessibilidade irrestrita.

Partindo destes pressupostos, neste trabalho foram verificados os índices de acessibilidade de repositórios digitais no Brasil, utilizando a ferramenta AccessMonitor. Considera-se que os resultados podem servir de orientação para que as instituições que criam e gerenciam repositórios informacionais, alcancem os parâmetros internacionais apontados pelo AccessMonitor, cumprindo desta maneira a legislação que garante direito de acessibilidade à educação, protagonizando iniciativas que se apoiam na ideia de que informações devem ser disponibilizadas para todos, sem qualquer espécie de barreira.

\section{REPOSITÓRIOS DIGITAIS E ACESSIBILIDADE}

Os repositórios digitais são bases de dados desenvolvidas para reunir, organizar e tornar acessível à produção científica de pesquisadores (LEITE et al., 2012). Ainda segundo os autores, sua principal finalidade é facilitar o acesso à produção de instituições específicas, podendo ser classificados como institucionais e temáticos. Os institucionais têm como objetivo agrupar a produção científica de determinada área ou tema (LEITE et al., 2012). Os repositórios também visam a preservação documental, servem como mecanismo de retroalimentação da produção e uso de informações, podendo contribuir no aumento da visibilidade, reconhecimento e prestígio das instituições (LEITE, 2009).

O Instituto Brasileiro de Informação em Ciência e Tecnologia - IBICT (2020) define repositórios digitais como

[...] bases de dados online que reúnem de maneira organizada a produção científica de uma instituição ou área temática. Os RDs armazenam arquivos de diversos formatos. Ainda, resultam em uma série de benefícios tanto para os pesquisadores quanto às instituições ou sociedades científicas, proporcionam maior visibilidade aos resultados de pesquisas e possibilitam a preservação da memória científica de sua instituição. Os RDs podem ser institucionais ou temáticos. Os repositórios institucionais lidam com a produção científica de uma determinada instituição. Os repositórios temáticos com a produção científica de uma determinada área, sem limites institucionais (IBICT, 2020).

Repositórios institucionais podem ser considerados como bibliotecas digitais, no entanto, nem toda biblioteca digital pode ser considerada um repositório institucional (LEITE et al., 2012). $\mathrm{Na}$ essência de ambos está a visibilidade e a vantagem da velocidade de disseminação. Além disso, ambos são sistemas de informação que trabalham com a organização e disponibilização de documentos digitais, porém, o foco de um repositório institucional é reunir, preservar e disponibilizar as informações produzidas no âmbito da instituição, as bibliotecas digitais não têm a função de preservação da memória institucional e costumam ter abrangência e finalidade mais amplas. 
Com a compreensão e o reconhecimento da importância da diversidade em todos os setores da sociedade, a ideia de acessibilidade se faz presente nas discussões e iniciativas de garantia de acesso e inclusão em todos os setores e lugares. Essa concepção inclui espaço físico, mobiliários, equipamentos urbanos, sistemas e meios de comunicação e informação (CORRÊA, 2009). Para Manzini (2005) o conceito de acessibilidade baseia-se em situações que podem ser vivenciadas nas condições específicas do dia a dia e que podem ser observadas, implementadas, medidas, reguladas e avaliadas. Desta forma, as condições de acessibilidade podem ser disponibilizadas para que todas as pessoas possam acessar espaços, vivenciar experiências, incluindo o uso de sistemas de informação (MANZINI, 2005). A Associação Brasileira de Normas Técnicas - ABNT (2015) define acessibilidade na norma NBR 9050, que afirma ser:

[...]a possibilidade e condição de alcance, percepção e entendimento para utilização, com segurança e autonomia, de espaços, mobiliários, equipamentos urbanos, edificações, transportes, informação e comunicação, inclusive seus sistemas e tecnologias, bem como outros serviços e instalações abertos ao público, de uso público ou privado de uso coletivo, tanto na zona urbana como na rural, por pessoa com deficiência ou mobilidade reduzida.

Em suma, podemos entender a acessibilidade como elemento que possibilita acesso igualitário a todas as coisas do mundo cotidiano incluindo os ambientes informacionais virtuais. As ferramentas de acessibilidade proporcionam aos usuários, de modo autônomo, toda a informação que lhe é apresentada, independentemente das suas características físicas, sem prejuízos quanto ao conteúdo da informação (TORRES; MAZZONI; ALVES, 2002). Ainda segundo Torres, Mazzoni e Alves (2002) esta acessibilidade é alcançada combinando a apresentação de informações de várias maneiras e com ferramentas que incluem, por exemplo, sistemas diferenciados para leitura de tela, reconhecimento visual ou sonoro de informações e simuladores de teclado.

É importante notar que a acessibilidade digital só pode ser viabilizada por meio da combinação entre hardwares e softwares, que disponibilizam mecanismos adequados para superar quaisquer espécies de barreiras de acesso à informação (PASSERINO; MONTARDO, 2007).

É relevante destacar a existência do consórcio Internacional World Wide Web (W3C), que tem como objetivo estimular o potencial da web desenvolvendo protocolos para promover o seu melhoramento e garantir sua operacionalidade (SILVA, 2012). Entre as principais áreas desenvolvidas pelo consórcio W3C é a Web Accessibility Initiative - WAI, constituída por um grupo de trabalho que além de realizar pesquisas de monitoramento relacionadas à acessibilidade, também elabora as Diretrizes de Acessibilidade para Conteúdo Web (WCAG)

Silva (2012) destaca que as páginas da web com recursos de acessibilidade podem aumentar a visibilidade e acesso ao site, além de promover a inclusão de pessoas com necessidades especiais, idosos e, em muitos casos, aqueles que não possuem recursos tecnológicos atualizados.

Assim, partindo do entendimento da relevância da acessibilidade no mundo virtual e que a função dos repositórios é disponibilizar informações de maneira ampla e irrestrita, os recursos de acessibilidade seriam inerentes e essenciais, sendo que os resultados deste trabalho poderão demonstrar se esta é uma afirmativa que corresponde à realidade.

\section{BREVE PANORAMA DE ESTUDOS SOBRE REPOSITÓRIOS E ACESSIBILIDADE}

$\mathrm{Na}$ literatura acadêmica brasileira o tema, acessibilidade em repositórios tem sido abordado sob múltiplas perspectivas, incluindo os aspectos da arquitetura de informação (MARQUES; VECHIATO, 2017); recursos específicos e necessários para acessibilidade em repositórios (CAMARGO; VIDOTTI, 2008); usabilidade (RIBEIRO; VIDOTTI, 2009); encontrabilidade (REIS et al., 2018); segurança (SANTOS; ARAÚJO, 2015); distintas tipologias documentárias como arquivos Ibero Americanos (PINTO; VIEIRA; BITENCOURT, 2019) e dados de pesquisas (SANCHEZ; VECHIATO; VIDOTTI, 2019), assim como estudos que consideram regiões geográficas, como de instituições de diferentes capitais brasileiras (SIEBRA; 
OLIVEIRA; MARCELINO, 2014); ou de algumas instituições (SANCHEZ; VECHIATO, 2017). O trabalho desenvolvido por Andrade, Lucas e Nascimento (2015) relata que as abordagens sobre deficiência visual e suas tecnologias assistivas predominam nos estudos sobre acessibilidade publicados em periódicos brasileiros. Vale destacar também a interessante proposta de Paula e Carvalho (2009) de criação, em cursos de graduação em biblioteconomia, de disciplina relacionada à acessibilidade de informações.

Sobre o conceito de usabilidade, Passerino e Montardo (2007) mencionam a importância de diferenciar da acessibilidade. A usabilidade de sistemas de informação, relacionada às questões de arquitetura de informação, se refere ao alcance satisfatório das expectativas dos usuários, no sentido de entender e perceber facilmente os recursos disponíveis, já a acessibilidade contempla o uso de interfaces especiais que proporcionam o acesso à informação para todos, sem exceções (PASSERINO; MONTARDO, 2007)

Entre as falhas de acessibilidade e recorrentes em repositórios Camargo e Vidotti (2008) apontam a necessidade de inclusão de teclas de atalhos, rotulagens de imagens, e a disponibilização de mapas dos websites.

Portanto, a partir deste breve panorama, que mostra a multiplicidade de estudos e abordagens, associados à importância da acessibilidade em ambientes e sistemas de informação, é possível inferir que os resultados da análise dos repositórios brasileiros deverá ser positiva. Após a descrição dos procedimentos metodológicos poderemos constatar se esta afirmativa está em consonância com a realidade.

\section{PROCEDIMENTOS METODOLÓGICOS}

A coleta de dados teve como ponto de partida a escolha dos repositórios institucionais cujo grau de acessibilidade seria analisado. Dessa forma, a opção foi utilizar o diretório de repositórios disponibilizado pelo Instituto Brasileiro de Informação em Ciência e Tecnologia IBICT. A lista exposta no site tem como fonte OASISBR, que é um portal brasileiro de publicações científicas em acesso aberto, cuja última atualização foi em 16 de agosto de 2019, durante o fechamento deste trabalho, uma vez que o IBICT se atualiza constantemente.

A coleta dos dados foi realizada no dia 31 de agosto de 2020, resultando em 109 repositórios. Destes, foram identificadas duas duplicatas e 14 não foram encontrados, isto é, as URLs não correspondiam ao repositório indicado ou encontram-se fora do ar.

Os dados dos 94 repositórios selecionados foram organizados em uma planilha para subsequente consulta das suas URLs no AccessMonitor. O AccessMonitor ${ }^{l}$ é uma ferramenta de verificação automática das Diretrizes de Acessibilidade para Conteúdo Web (WCAG) 2.0, do consórcio Internacional World Wide Web (W3C). A ferramenta é mantida pela Fundação para Ciência e Tecnologia de Portugal e recentemente foi atualizada para uma versão 2.1. As notas de acessibilidade variam entre 1 (baixa acessibilidade) e 10 (alta acessibilidade).

$\mathrm{O}$ Access Monitor indica erros de criticidade de acessibilidade, de acordo com as diretrizes internacionais, em três diferentes níveis: $\mathrm{A}$ - erros que indicam que aqueles websites não alcançam nível mínimo de acessibilidade; AA (ou duplo A) - sites acessíveis para a maioria de usuários; AAA (ou triplo A) - sites altamente acessíveis (GOVERNO ELETRÔNICO, [20--]). Estes níveis são determinados conforme critérios estipulados pelo W3C e que seguem três níveis de prioridades: Prioridade 1 - significa que os criadores de websites precisam respeitar critérios mínimos para que os usuários acessem as informações disponibilizadas; Prioridade 2 - são condições que os criadores de conteúdo para web devem cumprir para possibilitar que os usuários não tenham dificuldade de acesso às informações; Prioridade 3 - são pontos que os desenvolvedores podem utilizar para incrementar o nível de acessibilidade de seus websites. $\mathrm{O}$ AccessMonitor avalia 61 critérios nos websites, sendo 25 classificados como conformidade A (acessibilidade mínima), 13 de conformidade AA (acessibilidade satisfatória) e 23 no nível de conformidade AAA (excelente nível de acessibilidade). A ferramenta avalia como existente ou não existente, portanto, práticas de acessibilidade satisfatórias devem encontrar erros mínimos no nível A e AA.

\footnotetext{
${ }^{1}$ AccessMonitor: https://accessmonitor.acessibilidade.gov.pt/
} 


\section{ANÁLISE E DISCUSSÃO DOS RESULTADOS}

$\mathrm{Na}$ primeira etapa das análises foi possível observar a imprecisão existente na nomenclatura dos repositórios, pois muitos utilizam, inadequadamente, títulos diversos, incluindo biblioteca digital, biblioteca virtual, biblioteca multimídia, banco digital ou simplesmente acervo. Sendo assim, a primeira recomendação deste trabalho seria o uso adequado para definição dos sistemas informacionais. Com certeza essa ambiguidade resulta em equívocos desnecessários. É válido destacar a necessidade de averiguar a origem deste problema, isto é, se o IBICT reuniu em um mesmo diretório, bibliotecas digitais e repositórios ou se as próprias instituições utilizaram denominações inadequadas. Entretanto, como este não era o propósito deste trabalho, esta averiguação não foi realizada.

As notas determinadas pelo Access Monitor, conforme os critérios de acessibilidade dos websites, variam entre zero e dez, sendo que a média geral obtida pelos repositórios brasileiros analisados foi cinco. Entre os 94 analisados, 32 receberam nota cinco. No Gráfico 1 é possível verificar que somente dois repositórios obtiveram notas acima de sete, sendo que os dois são repositórios universitários: PUC-RS que alcançou nota oito e Universidade Federal dos Vales do Jequitinhonha e Mucuri (MG) que obteve nota nove. Entre os 94 repositórios avaliados, 63 (67\%) obtiveram nota inferior a cinco. Portanto, fica evidente que os responsáveis pelos repositórios brasileiros precisam ficar mais atentos aos recursos de acessibilidade. A lista completa dos repositórios e as notas obtidas pelo AccessMonitor estão disponibilizadas em uma tabela suplementar deste artigo

Gráfico 1 - Distribuição do número de repositórios brasileiros conforme nota obtida no

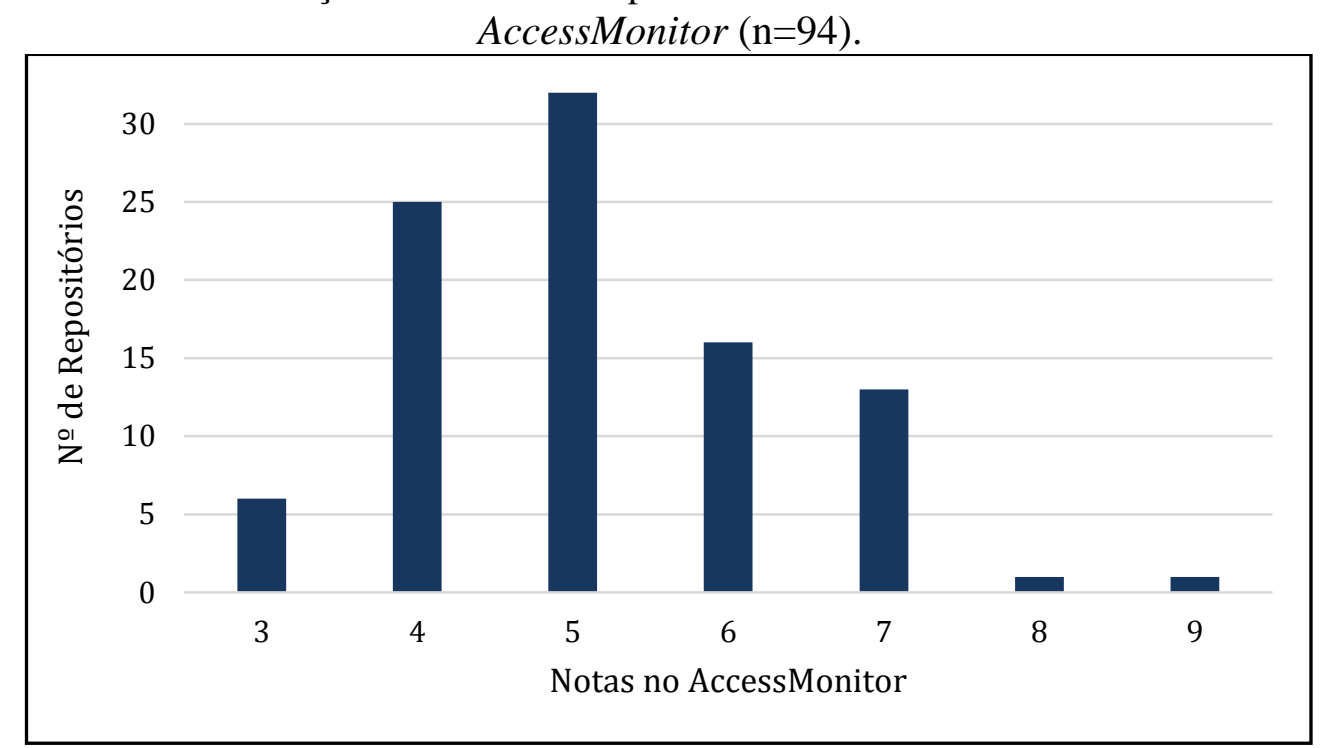

Fonte: Os autores

No que se refere aos critérios (A, duplo A e triplo A) verificou-se que todos os repositórios analisados apresentaram erros de nível $\mathrm{A}$, portanto não alcançaram critérios mínimos de acessibilidade. 56 entre apresentaram erros de nível AA e 82 de AAA.

O Gráfico 2, abaixo, mostra a quantidade de erros identificados nos repositórios conforme o nível de conformidade. 
Gráfico 2 - Distribuição da quantidade de erros conforme os níveis de conformidade do AccessMonitor ( $\mathrm{n}=94)$.

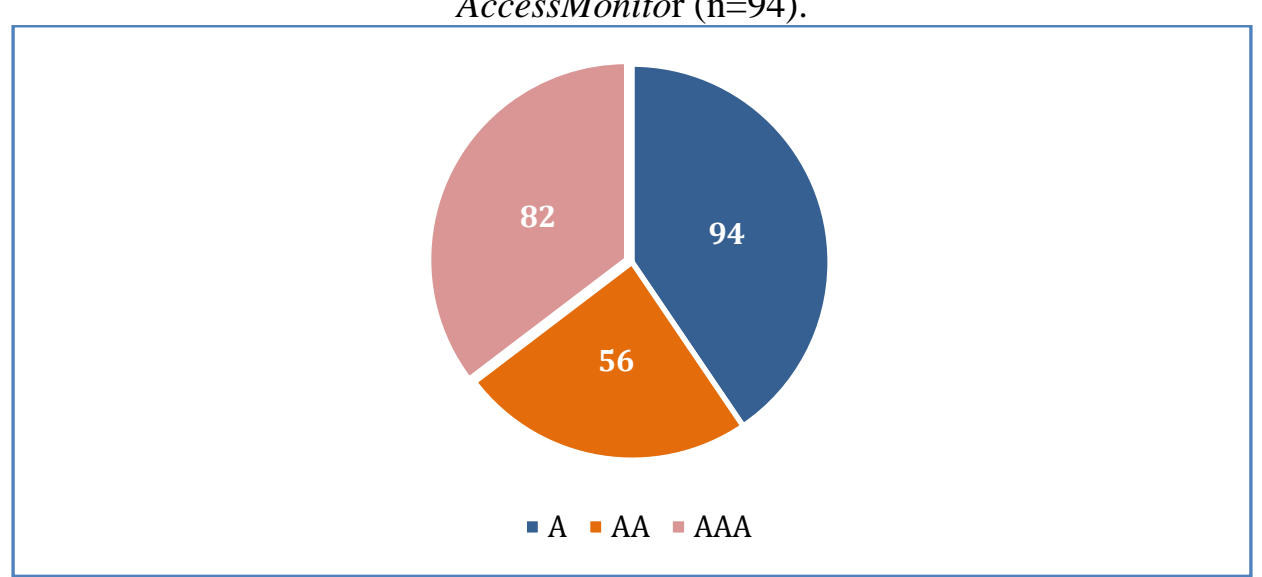

Fonte: Autores.

Também foram identificadas as médias de erros conforme cada nível, sendo que a média foi 14 e na tabela abaixo. No nível A, média de erros foi 11; no nível AA a média de erro foi um e no nível de conformidade triplo A (AAA) a média de erros foi de dois. Por fim, optou-se em identificar os repositórios que alcançaram critérios abaixo das médias na nota dada pelo Access Monitor, assim como nos níveis de conformidade de acessibilidade. A Tabela 1 mostra os três repositórios com menor nível de acessibilidade.

Tabela 1 - Repositórios com menores níveis de acessibilidade.

\begin{tabular}{lcccc}
\hline \multicolumn{1}{c}{ Repositório } & Nota & $\begin{array}{c}\text { Erros de } \\
\text { Nível A }\end{array}$ & $\begin{array}{c}\text { Erros de } \\
\text { Nível AA }\end{array}$ & $\begin{array}{c}\text { Erros de } \\
\text { Nível AAA }\end{array}$ \\
\hline $\begin{array}{l}\text { CBPF Index - Centro Brasileiro de Pesquisas } \\
\text { Físicas }\end{array}$ & 2,9 & 15 & 2 & 4 \\
$\begin{array}{l}\text { Repositório Institucional Pantheon da } \\
\text { Universidade Federal do Rio de Janeiro }\end{array}$ & 4,1 & 15 & 3 & 3 \\
$\begin{array}{l}\text { Repositório Digital da Universidade } \\
\text { Municipal de São Caetano do Sul }\end{array}$ & 4,2 & 14 & 2 & 3 \\
\hline
\end{tabular}

Fonte: Os autores.

\section{CONSIDERAÇÕES FINAIS}

No decorrer desta investigação foi evidenciado que repositórios governamentais e universitários que deveriam ser referência nacional, não estão exercendo esta função, considerando que estão na faixa amarela precisando de cuidados. Assim sendo, a criação dos repositórios digitais não está contemplando plenamente a diversidade de usuários, isto é, não está facilitando o acesso à informação para os usuários com deficiências, incluindo cegueira e baixa visão, surdez e baixa audição, dificuldades de aprendizagem, limitações cognitivas, limitações de movimentos, incapacidade de falar, fotossensibilidade e combinações destas características.

A pesquisa deixa como sugestão, para uma melhor elaboração e ajustes desses repositórios, uma orientação com quem realmente utiliza os recursos de acessibilidade tendo em vista que eles sabem identificar as falhas que estes espaços podem apresentar. Portanto, é importante incluir portadores de necessidades especiais nas equipes que testam os ambientes digitais de qualquer instituição. 


\section{REFERÊNCIAS}

ANDRADE, S.; LUCAS, E.R.O.; NASCIMENTO, M.J. Acessibilidade para usuários da informação com deficiência: um estudo de artigos em Biblioteconomia e Ciência da Informação. Biblionline, João Pessoa, v. 11, n. 1, p. 1-20, 2015. Disponível em: https://periodicos.ufpb.br/ojs2/index.php/biblio/article/view/24550. Acesso em: 1 set. 2020.

ASSOCIAÇÃO BRASILEIRA DE NORMAS TÉCNICAS. NBR 9050: acessibilidade a edificações, mobiliário, espaços e equipamentos urbanos. Rio de Janeiro: ABNT, 2004.

BERRÍO-ZAPATA, C.; SANTOS, Z.E.C.; CHALHUB, T. Acessibilidade digital na perspectiva dos cidadãos com deficiências: evolução e desafios. In: NUNES, Martha Suzana Cabral (org.). Desafios da inclusão na práxis pedagógica: saberes e fazeres em ciência da informação. São Paulo: ABECIN, 2020. cap. 5, p. 93-121. Disponível em: https://livroaberto.ufpa.br/jspui/handle/prefix/818. Acesso em: 1 set. 2020.

CAMARGO, L.S.A; VIDOTTI, S.B.G. Uma estratégia de avaliação em repositórios digitais. In: SEMINÁRIO NACIONAL DE BIBLIOTECAS UNIVERSITÁRIAS, 15., 2008, São Paulo. Anais [...]. São Paulo: CRUESP, 2008. Disponível em: http://repositorio.febab.libertar.org/temp/snbu/SNBU2008_007.pdf. Acesso em: 1 set. 2020.

CAMARGO, L.S.A.; VIDOTTI, S; APARECIDA, B.G. Arquitetura da informação para ambientes informacionais digitais. In: ENCONTRO NACIONAL DE PESQUISA EM CIÊNCIA DA INFORMAÇÃ̂, 9., 2008, São Paulo. Anais [...]. São Paulo: USP, 2008. Disponível em: http://200.20.0.78/repositorios/handle/123456789/1902?show=full. Acesso em: 26 set. 2020.

CORRÊA, P.M. Acessibilidade: Conceitos e Formas de Garantia. Revista Brasileira de Educação Especial, Marília, 2009. Disponível em:

https://www.scielo.br/scielo.php?pid=S1413-65382009000100012\&script=sci_arttext. Acesso em: 1 set. 2020.

GOVERNO ELETRÔNICO. Recomendações de acessibilidade: WCAG 2.0. [20--].

Disponível em: http://emag.governoeletronico.gov.br/cursoconteudista/desenvolvimentoweb/recomendacoes-de-acessibilidade-wcag2.html. Acesso em: 8 nov. 2020.

HOTT, D.F.M.; CRUZ-RIASCOS, S.A. Ciência da informação e interações teórico-sistêmicas com a acessibilidade. In: Encontro Nacional de Pesquisa em Ciência da Informação, 19., 2018, Londrina. Anais [...]. Londrina: Programa de Pós-Graduação em Ciência da Informação da Universidade Estadual de Londrina (PPGCI/UEL), 2018. Disponível em: https://repositorio.unb.br/handle/10482/33027. Acesso em: 1 set. 2020.

LEITE, F.; AMARO, B.; BATISTA, T.; COSTA, M. Repositórios institucionais: boas práticas para a construção de repositórios institucionais da produção científica. Brasília: IBICT, 2012. Disponível em: http://livroaberto.ibict.br/handle/1/703. Acesso em: 28 ago. 2020.

LEITE, F.C.L. Como gerenciar e ampliar a visibilidade da informação científica brasileira: repositórios institucionais de acesso aberto. Brasília: IBICT, 2009.

MANZINI, E.J. Inclusão e Acessibilidade. Revista da Sobama, Marília, 2005. Disponível em: http://fio.edu.br/site2013/images/NAU/001d-\%20manzini\%202005\%20acessibilidade.pdf. Acesso em: 1 set. 2020. 
MARQUES, C.A.G.; VECHIATO, F.L. Arquitetura da informação em repositórios digitais: análise do repositório institucional da universidade federal do rio grande do norte. Bibliocanto, v. 3, n. 1, n. 1, p. 2-28, 2017. Disponível em:

https://periodicos.ufrn.br/bibliocanto/article/view/11944. Acesso em: 26 set. 2020.

PASSERINO, L.M.; MONTARDO, S.P. Inclusão social via acessibilidade digital: Proposta de inclusão digital para Pessoas com Necessidades Especiais. E-Compós, Brasília, v. 8, 2007. Disponível em: https://www.e-compos.org.br/e-compos/article/view/144. Acesso em: 1 set. 2020.

PAULA, S.N.; CARVALHO, J.O.F. Acessibilidade à informação: proposta de uma disciplina para cursos de graduação na área de biblioteconomia. Ci. Inf, Brasília, v. 38, n. 3, p. 64-79, 2009. Disponível em http://www.scielo.br/scielo.php?script=sci_arttext\&pid=S0100$19652009000300005 \& \operatorname{lng}=$ pt\&nrm=iso. Acesso em 26 set. 2020.

PINTO, A.F.A.; VIEIRA, T.O.; BITTENCOURT, P.R. Acessibilidade informacional na web: um estudo da acessibilidade nas instituições arquivísticas nacionais da Ibero-América. Páginas a\&b, s. 3, n. 12, 2019. Disponível em:

http://ojs.letras.up.pt/index.php/paginasaeb/article/view/6363. Acesso em: 26 set. 2020.

REIS, S.G.O. et al. Avaliação da encontrabilidade da informação do repositório institucional da universidade tecnológica federal do Paraná. InCID: Revista de Ciência da Informação e Documentação, v. 9, n. 2, n. 2, p. 169-188, 2018. Disponível em: https://www.revistas.usp.br/incid/article/view/126124. Acesso em: 26 set. 2020.

RIBEIRO, O.B.; VIDOTTI, S.A.B.G. Otimização do acesso à informação científica: discussão sobre a aplicação de elementos da arquitetura da informação em repositórios digitais. BIBLOS Revista do Instituto de Ciências Humanas e da Informação, v. 23, n. 2, p. 105-116, 2009. Disponível em: https://www.seer.furg.br/biblos/article/view/1309. Acesso em: 26 set. 2020.

SANCHEZ, F.A.; VECHIATO, F.L. Encontrabilidade da informação em repositórios digitais: um enfoque nos repositórios institucionais da USP, Unesp e Unicamp. In: Encontro Nacional de Pesquisa em Ciência da Informação, 18, 2017, Marília. Anais [...]. Marília: UNESP, 2017. p. $\quad$ ENANCIB, 2017. Disponível em: https://brapci.inf.br/index.php/res/v/104846. Acesso em: 26 set. 2020.

SANCHEZ, F.A.; VECHIATO, F.L.; VIDOTTI, S.A.B.G. Encontrabilidade da informação em repositórios de dados: uma análise do dataone. Informação \& Informação, v. 24, n. 1, p. 5179, 2019. Disponível em:

http://www.uel.br/revistas/uel/index.php/informacao/article/view/30725. Acesso em: 26 set. 2020.

SANTOS, C.G.D.; ARAÚJO, W.J. Acessibilidade informacional: um estudo sobre configurações de segurança em objetos digitais acessíveis segundo análise de aceitação por pessoas com deficiência visual. Pesquisa Brasileira em Ciência da Informação e Biblioteconomia, v. 10, n. 2, 2015. Disponível em: https://periodicos.ufpb.br/ojs/index.php/pbcib/article/view/26227. Acesso em: 26 set. 2020.

SIEBRA, S.A.; OLIVEIRA, J.N.N.; MARCELINO, C.S. Avaliação do acesso e visualização da informação em repositórios institucionais. Informação \& Tecnologia, v. 1, n. 2, p. 77-95, 2014. Disponível em: https://periodicos.ufpb.br/index.php/itec/article/view/21535. Acesso em: 26 set. 2020. 
SILVA, S. Acessibilidade Digital em Ambientes Virtuais de Aprendizagem. Geinte, São Cristóvão, v. 2, n. 3, p. 245-254, 2012. Disponível em:

http://revistageintec.net/index.php/revista/article/view/48/108. Acesso em: 1 set. 2020.

SOUZA, M.R.F.; GUIMARÃES, Í.J.B. Acessibilidade nos mecanismos de busca dos periódicos brasileiros em ciência da informação. Tendências da Pesquisa Brasileira em Ciência da Informação, 2015. Disponível em: https://brapci.inf.br/index.php/res/v/119567. Acesso em: 1 set. 2020 .

TORRES, E.F.; MAZZONI, A.A.; ALVES, J.B.M. A acessibilidade à informação no espaço digital. Ciência da Informação, Brasília, v. 31, n. 3, 2002. Disponível em:

https://www.scielo.br/scielo.php?script=sci_arttext\&pid=S010019652002000300009\&lng=pt\&tlng=pt. Acesso em: 1 set. 2020. 